\title{
RESPONSE TO EDITORIALS Saccular aneurysms in the Barrow Ruptured Aneurysm Trial: 10 years later
}

\author{
Robert F. Spetzler, MD, Felipe C. Albuquerque, MD, Joseph M. Zabramski, MD, and \\ Peter Nakaji, MD
}

\author{
Department of Neurosurgery, Barrow Neurological Institute, St. Joseph's Hospital and Medical Center, Phoenix, Arizona
}

$\mathrm{W}$ E thank Drs. Riina and Barker, Dr. Cockroft, and Dr. Amenta and colleagues for their remarks. We will address each in turn.

We appreciate the nice summary of our publication by Drs. Riina and Barker. However, we believe that it is irrelevant whether a stent is added or whether clinicians have strong feelings about the right treatment in the absence of supporting data.

We applaud Dr. Cockroft on his erudite and elegant discourse on the results of the Barrow Ruptured Aneurysm Trial (BRAT). Dr. Cockroft, arguing for a "more nuanced view of individualized care based on numerous factors," suggests that practitioners have some nonvalidated insight that gives them the ability to determine best practice. Although the art of medicine gives us leeway in recommending to our patients what we believe is in their best interest, randomized trials remain the gold standard to establish "best practice."

Dr. Cockroft states, "Unfortunately, everyone dies eventually, so when death is part of your primary outcome measure, if you wait long enough your outcomes will converge. In the case of BRAT, we know of only 2 deaths related to aneurysms (and one of these was not even from the target aneurysm). Although both were in the coil-assigned and -treated group, these deaths are overwhelmed by deaths from other causes that constitute the major reason for the increasing poor outcomes over time."

Naturally, it is true that everyone eventually dies, but it is equally true that the sole purpose of treating these patients is to prevent further hemorrhage and, in both the BRAT and the International Subarachnoid Aneurysm Trial (ISAT), this purpose is best accomplished with clipping. For the patient, it makes no difference whether they bleed from the previously ruptured target aneurysm or from an additional incidentally treated aneurysm.

A significant portion of Dr. Cockroft's comments relate to the 1-year results, in which a benefit was demonstrated for the entire intent-to-coil cohort; this was not the case for later time points. Due to the senior author's naïveté, we did not predetermine the appropriate cohorts to be studied, but the fact remains that it was always our intent to compare our results with those of the ISAT. Thus, when one considers the results of the ISAT, in which the patients overwhelmingly had small, low-grade, anterior circulation aneurysms, it was our fault for not prespecifying these characteristics for the cohorts that we wanted to study. For the sake of completeness, data on the actually treated saccular anterior circulation aneurysms are included in Table 1.

These data demonstrate not only that the assigned cohorts were the same as in our publication but also that the actually treated aneurysms had similar outcomes. Despite the inclusion of more-difficult aneurysms that were assigned to endovascular coiling but were actually treated with clipping, plus those actually treated with clipping because of large hematomas, the results demonstrate a slight, although statistically nonsignificant, benefit to clipping. Thus, from a pool of 314 randomized patients, all those randomized to undergo clipping (with the exception of a single patient) had their aneurysms actually clipped, and in addition, $36 \%$ of cross-over patients who were randomized to undergo coiling had their aneurysms actually clipped. This finding emphasizes that the use of clipping to treat all anterior circulation aneurysms could achieve results equal to those for the selected anterior circulation coiling treatment cohort.

The lack of middle cerebral artery (MCA) aneurysms in the cohorts treated with coiling in both ISAT and BRAT leaves the question of the best treatment for these aneurysms open.

The final question that Dr. Cockroft poses is, "then this would suggest that if you have your ruptured aneurysm treated with open craniotomy and clipping by arguably one of the best cerebrovascular surgeons in the country, if not the world, at a facility known for its surgical prowess, 
TABLE 1. Patients with saccular anterior circulation aneurysms with an $\mathrm{mRS}$ score $>2$, grouped by actual treatment with coiling or clipping

\begin{tabular}{|c|c|c|c|c|}
\hline \multirow[b]{2}{*}{$\begin{array}{l}\text { Duration of } \\
\text { Follow-Up }\end{array}$} & \multirow{2}{*}{$\begin{array}{l}\text { No. of Pts } \\
\text { Available for } \\
\text { Analysis }\end{array}$} & \multicolumn{2}{|c|}{ No. $(\%)$ of Pts } & \multirow[b]{2}{*}{$\begin{array}{c}p \\
\text { Value }\end{array}$} \\
\hline & & $\begin{array}{c}\text { Treated w/ } \\
\text { Coiling }\end{array}$ & $\begin{array}{l}\text { Treated w/ } \\
\text { Clipping }\end{array}$ & \\
\hline $1 \mathrm{yr}$ & 273 & 18/87 (21) & $55 / 186(30)$ & 0.12 \\
\hline 3 yrs & 263 & $21 / 84(25)$ & $58 / 179(32)$ & 0.22 \\
\hline 6 yrs & 251 & $24 / 78(31)$ & 67/173 (39) & 0.23 \\
\hline $10 \mathrm{yrs}$ & 221 & $36 / 69(52)$ & $75 / 152(49)$ & 0.70 \\
\hline 10 yrs w/ CF* & 280 & $39 / 89(44)$ & 83/191 (44) & 0.95 \\
\hline \multicolumn{5}{|l|}{ Deaths } \\
\hline $10 \mathrm{yrs}$ & 221 & $25 / 69(36)$ & $45 / 152(30)$ & 0.33 \\
\hline 10 yrs w/ CF* & 280 & $25 / 89(28)$ & 45/191 (24) & 0.42 \\
\hline
\end{tabular}

$\mathrm{CF}=$ carried forward; $\mathrm{pts}=$ patients.

* Includes patients seen at 1-, 3-, or 6-year follow-up but not at 10-year follow-up.

your best hope at 10 years is that you will have the same chance of a poor outcome as someone who had their aneurysm coiled with what is now 15-year-old technology. If that is the case, then why in this day and age would anyone choose to have their aneurysm clipped?"

We reply that all aneurysms in the anterior circulation that are treated with clipping are associated with the same risk; that clipping, unlike coiling, does not require retreatment in almost $20 \%$ of cases; that clipping is associated with much better long-term occlusion rates than coiling; and finally, that the risks of bleeding and death from rerupture associated with clipping are statistically superior to those associated with coiling.

Finally, we appreciate the thoughtful points raised by Dr. Amenta and colleagues. We have replied to individual issues within the text of the editorial to provide the readers with the context for our reply.

Amenta et al. point out that "at many centers throughout the world, $70 \%-90 \%$ (or higher) of patients harboring ruptured aneurysms are treated with endovascular methods" and argue that "as a result, evaluation of the BRAT data in the setting of the present environment can be difficult, particularly in regard to the disparity in cross-overs between the groups."

One cannot have it both ways. Because there is no allinclusive prospective study comparing these two treatments, it is irrelevant that there are many centers that use endovascular therapy for more than $90 \%$ of the aneurysms that they treat. If we wish to base our treatment on highlevel prospective studies, then we are woefully lacking these data. This phenomenon occurs against the backdrop of a 36\% cross-over in BRAT by two excellent endovascular practitioners and a selected cohort of approximately $20 \%$ small anterior circulation aneurysms studied in ISAT. There has been no trial to demonstrate that, for example, MCA aneurysms should be treated endovascularly. Endovascular treatment of MCA aneurysms is underrepresented in both of these studies, but such procedures are clearly performed routinely in centers where more than $90 \%$ of aneurysms are treated endovascularly.

Amenta et al. contend that "it should be taken into con- sideration that only a single patient crossed over from the clip-assigned group to the coiling group. Not only is this disparity striking from a statistical perspective, it also probably does not accurately reflect current practice or the way in which IA [intracranial aneurysm] management is now evaluated. Is a ruptured wide-necked ophthalmic aneurysm in a patient with normal vision really better managed with clipping and the surgical adjuncts of clinoid removal and cervical carotid exposure? Many would probably argue that these lesions are more suitable for balloon-assisted coiling. A contemporary study may have considerably less cross-over from the coil-to-clip group and significantly greater cross-over from the clip-to-coil group."

We do not see how the fact that there was one crossover case in which a patient who was assigned to treatment with clipping was actually treated with coiling is a statistical problem. Before the introduction of coiling, these aneurysms, when treated, were all treated surgically. To evaluate whether this treatment is appropriate in comparison to coiling was the point of the study. We emphasize that the goal of a randomized trial is not to determine current practice; rather it is to provide meaningful data to influence current practice. These studies are indeed designed to provide treatment guidance regarding the question raised by the example of a ruptured wide-necked ophthalmic aneurysm, not to argue for a particular treatment.

Amenta et al. point out that we have examined only patients with ruptured saccular intracranial aneurysms and argue that this introduces potential problems to the analysis. They highlight that our analysis is of a subgroup that was not predefined in the trial design. Second, they note that the definition of saccular versus nonsaccular aneurysm can be subjective and introduces potential bias. They state that when we compared the analysis of patients harboring saccular aneurysms versus all types of aneurysms with 6 years of follow-up, "for posterior circulation lesions coiling was associated with better outcomes if all aneurysms were included, whereas no significant differences in outcomes were observed when analyzing only saccular aneurysms. It is not clear if such differences in outcome would be seen at 10 years if all patients with aneurysms were included in this analysis."

These are good, pertinent points, and we had three experts who were not involved in treating study patients evaluate the initial designation of aneurysm type to obtain the greatest fidelity. Although not reported in this publication, which was restricted to saccular aneurysms for comparison to ISAT, we found that there was no statistical difference at any time point after our initial 1-year publication when the entire cohort was included (see Table 2).

Amenta et al. remark that we "do not include a table examining outcomes in patients with saccular aneurysms by actual treatment." The reason for not including the actual treatment results is that they are meaningless in an intent-to-treat trial, especially with a $36 \%$ cross-over rate. Also, additional tables would have exceeded the number allowed by the Journal of Neurosurgery. However, there is no statistically significant difference in clinical outcome or deaths when comparing the actual treatments performed. For transparency and for comparison with ISAT, 
TABLE 2. Patients with mRS score $>2$ across BRAT follow-up grouped by assigned treatment

\begin{tabular}{|c|c|c|c|c|c|c|}
\hline \multirow[b]{2}{*}{ Duration of Follow-Up } & \multirow[b]{2}{*}{ No. of Pts Available for Analysis } & \multicolumn{2}{|c|}{ Assigned Treatment, No. (\%) of Pts } & \multirow[b]{2}{*}{ OR } & \multirow[b]{2}{*}{$95 \% \mathrm{Cl}$} & \multirow[b]{2}{*}{$\mathrm{p}$ Value } \\
\hline & & Coiling & Clipping & & & \\
\hline $1 \mathrm{yr}$ & 358 & $42 / 174(24.1)$ & $64 / 184(34.8)$ & 1.68 & $1.06,2.67$ & 0.03 \\
\hline 3 yrs & 349 & $51 / 170(30.0)$ & $64 / 179(35.8)$ & 1.30 & $0.83,2.04$ & 0.25 \\
\hline 3 yrs w/ CF* & 366 & $51 / 178(28.7)$ & $64 / 188(34.0)$ & 1.29 & $0.83,2.00$ & 0.27 \\
\hline 6 yrs & 336 & $57 / 162(35.2)$ & $72 / 174(41.4)$ & 1.30 & $0.84,2.02$ & 0.24 \\
\hline 6 yrs w/ CF† & 365 & $60 / 177(33.9)$ & 73/188 (38.8) & 1.24 & $0.81,1.90$ & 0.33 \\
\hline 10 yrs & 299 & $71 / 143(49.7)$ & $81 / 156(51.9)$ & 1.10 & $0.70,1.73$ & 0.69 \\
\hline 10 yrs w/ CF & 369 & $78 / 179(43.6)$ & $88 / 190(46.3)$ & 1.12 & $0.74,1.69$ & 0.60 \\
\hline
\end{tabular}

data for the actual treatments performed and deaths by actual treatment performed for anterior circulation saccular aneurysms are included in Table 1.

As Amenta et al. point out, "Patients who were assigned to coiling and underwent clipping had a trend toward worse outcomes at all time points. It should be considered that these IAs not deemed suitable for endovascular therapy may represent a more complex subset of lesions (i.e., wide neck, incorporation of parent vessel, large size) or be associated with hemorrhage requiring evacuation, thus making treatment by any modality inherently more difficult and prone to complication or inherently associated with an increased probability of poor outcome. This, of course, would negatively impact the outcome in those crossing over from coiling to clipping."

Naturally, those patients who crossed over from being assigned to receive treatment with coiling to actually receive treatment with clipping for urgent associated hematoma evacuation had a much worse prognosis than other patients in the study. This makes the point that actual treatment tables are irrelevant. However, it should be noted that despite the cross-overs, when actual treatments are compared at 10 years of follow-up, the absolute percentages are nearly identical and slightly favor clipping, although this difference is not statistically significant.

https://thejns.org/doi/abs/10.3171/2018.12.JNS182853

\section{Acknowledgments}

Funding for this study was provided by the Barrow Neurological Foundation and the Hanley Aneurysm Fund. We thank the staff of Neuroscience Publications at Barrow Neurological Institute for assistance with manuscript preparation.

\section{Disclosures}

Dr. Spetzler is a consultant for Carl Zeiss Meditec, AG, and he receives royalties from Stryker Corp. and Kogent Surgical. Dr. Nakaji is a consultant for Carl Zeiss Meditec and a holder of GT Medical Technologies stock.

\section{Correspondence}

Robert F. Spetzler: neuropub@barrowneuro.org.

\section{INCLUDE WHEN CITING}

Published online March 8, 2019; DOI: 10.3171/2018.12.JNS182853. 